
Military Technical College Kobry El-Kobbah, Cairo, Egypt.

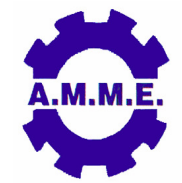

$15^{\text {th }}$ International Conference on Applied Mechanics and Mechanical Engineering.

\title{
STUDYING THE PROCESS OF OBTAINING FINE COPPER POWDERS BY ELECTROLYSIS
}

\author{
R. S. Akpanbayev*
}

\begin{abstract}
The electrolytic reception of copper powders is the most studied and widely applied method, but at the implementation of process of electro-deposition, it is difficult to obtain fine and ultrafine powders.

Surveys have established technological regimes of electrolysis (cathodic current density, copper and sulfuric acid concentrations, duration of electrolysis, etc.). The current efficiency depending on the cathode material was studied.

The influences of organic compounds on dendrite-formation, size and grain shape of obtained copper powder are studied. The nature of organic additives with different functional groups and structure, affect the nature of grit and dispersion and the crystal structure of copper deposits, and causes reflexion in color transition of the deposited powder.
\end{abstract}

\section{KEY WORDS}

Copper powder, Electrolysis, Electrolyte, Micrographs, Cathodes.

\footnotetext{
* PhD candidate, Dept. of Metallurgical Processes, Thermal Engineering and Technology of Special Materials, KazNTU, Almaty, Kazakhstan.
} 


\section{INTRODUCTION}

Scopes of application of copper powders are varied and they are constantly expanding. Among the priority areas for the use of copper powder production are nano functional materials, miniaturization of components in communications technology and electronics, production of precision alloys with unique technical characteristics, composite materials for various purposes, etc.

High demands for copper powders industries requires new and improve existing technologies of their production to increase the dispersion of the powder and to increase its purity, as well as the intensification of the process.

Among the methods of preparation of copper powders most studied and widely used is the electrolytic method, but the implementation process of electro-deposition of metals is difficult to obtain fine and ultrafine powders.

The electrode position of copper powders, as known, proceeds with the formation of dendritic crystal particles, the average size of which depend on the electrolyte composition, temperature, duration of electrolysis, etc. Thus, with increasing current density of the process into the realm of the ever-increasing difficulty of diffusion: increasing polarization, contributing to the formation of many new centers of crystallization [1, 2], which leads to increased dispersion of the formed particles and dendritic deposits. With increasing concentration of copper ions increases the limiting current density. Temperature affects not only the value of the limiting current, but also on the rate of nucleation and crystal growth, passivation of the faces of growing crystals, and other factors of electrolysis, which differently vary with temperature [3].

\section{SULFURIC ACID INFLUENCE}

Influence of sulfuric acid concentration on copper current efficiency and output of copper powder small fraction is reflected in Fig. 1. Conditions for research: the current density nearby $1000 \mathrm{~A} / \mathrm{m}^{2}$, copper concentration in the electrolyte $10 \mathrm{~g} / \mathrm{l}$ and duration of $10 \mathrm{~min}$, electrolysis was performed using a copper cathode. On the basis of the received data it is possible to draw a conclusion, that with increase of concentration of acid the current efficiency increases constantly, and the yield of fines - up to a concentration of acid in electrolyte $170 \mathrm{~g} / \mathrm{l}$.

\section{STUDYING THE CATHODE MATERIALS}

By consideration of cathodes material influence (Fig. 2) observed the highest copper current efficiency and an exit of small fraction at use of the graphite cathode that is connected with its superficial and redox properties.

The lowest current efficiency, as well as the output of fines, is observed for all other things being equal on a steel cathode, which may be associated with an active release of hydrogen on it, and a simultaneous growth on it of an overstrain. 
Qualitative analysis of copper powder showed that using a graphite cathode greatly increases the proportion of carbon in powder, which could adversely affect the properties of final products, therefore concluded that, despite the high current efficiency and yield of fines on a graphite cathode, its use is inappropriate.

Thus, on the basis of the received data it is possible to recommend for reception of a copper powder of fraction less than 75 microns at duration of process of 10 minutes to use the copper cathode.

As a result of research, the following technological modes of electrolysis are established: cathode current density $750-820 \mathrm{~A} / \mathrm{m}^{2}$, concentrations in the electrolyte of sulfuric acid - 30-50 g/l and copper - 5-20 g/l, the duration of electrolysis to remove the powder - not more than 10 minutes, the temperature of the electrolyte $-25-30^{\circ}$. The most efficient and quality of the copper powder deposition occurred on the copper and graphite cathodes.

\section{INFLUENCE OF ORGANIC COMPOUNDS}

As for the electrolytic deposition used organic additives, further attempts to identify their influence on the formation of copper deposits and their change in tone color is made. At carrying out of a series of experiments with the solutions containing the additives of organic substances, observed allocation of different amounts of hydrogen, which depended on the nature of organic additive, and the magnitude of the cathodic current density. As the organic additives used acetic acid and citric acid.

Electron microscopic analysis of the powders showed that the samples of copper from solutions containing additives of organic acids differ from the micrographs of the copper powder obtained from the solution without organic additives (Fig. 3). The surface of the copper powder, extracted from the sulfuric acid electrolyte is denser than copper obtained in the presence of organic reagents.

Dispersity of copper powder increases with the addition in electrolyte of citric acid solution. Thus on the surface of copper powder, there are medium-sized units, which have a spherical shape. At the same time, an additive in the electrolyte of citric acid under the same conditions of electrolysis in quantity of $\geq 1 \mathrm{~g} / \mathrm{l}$ caused formation on the cathode, a dense shiny copper foil, badly separated from the base.

The addition of even small quantities of acetic acid in electrolyte caused reception of a coarse-grained deposit with the expressed crystal structure outwardly similar to small metal sawdust. Increasing the concentration of acetic acid in the electrolyte increases the crystallinity and granularity of a deposit. Besides, the structure of a copper powder gets even more ordered character.

It is revealed that the injection of organic compounds in an electrochemical system has an essential impact on dendrite-formation, size and shape of grains deposited copper powder. Moreover, the nature of organic additives with different functional groups and structure, influencing the nature of the grain, dispersion and crystal structure of powder is also reflected in the color transition of the deposited powder. 
In the case of copper deposition without the organic phase fairly dense dark brown copper powder with small patches of dark-red color is formed. In the process beginning observed the intensive allocation of hydrogen stopping with development of an electrode surface at the expense of copper deposition.

Significant influence on the color change of deposit provides an injection of citric acid to the electrolyte. Color of deposit varies from dark brown to reddish-brown, due to their greater dispersion than previous samples.

The addition of acetic acid in the electrolyte allows keeping a bright tone color of copper powder. The resulting deposit is red with an orange shade. Change in tone color associated with an increased dispersion of the deposit and the crystal's structure.

On the basis of the received data it is possible to draw a conclusion, that with increase of concentration of acid the current efficiency and the yield of fines increases constantly up to a concentration of acid in electrolyte $170 \mathrm{~g} / \mathrm{l}$. The highest copper current efficiency and an exit of small fraction observed at use of the graphite cathode that is connected with its superficial and redox properties, but to receive copper powder with high purity recommended using of copper cathode. Dispersity of copper powder increases with the addition in electrolyte of citric acid solution, thus there are aggregates, which have a spherical shape. The addition of acetic acid in electrolyte caused reception of a coarse-grained deposit with the expressed crystal structure. The injection of organic compounds in an electrochemical system has an essential impact on dendrite-formation, size, and shape of grains and coloring of deposited copper powder.

Thus, implementing new approaches to finding the optimum electrolyte composition and method of electrolysis can be solved extremely relevant scientific and practical problem - obtaining fine powders of metals, particularly copper.

\section{REFERENCES}

[1] Hamburg, U.D., The dependence of the grain size of electrochemically deposited metal against overvoltage, Electrochemistry, No.9, pp 1157-1159, (1999).

[2] Kobzhanov, A.S., Kurbatov, A.P., Romanov, G.A., Influence of cathode current density on the deposition of copper powders., Messenger of KazNU, Chemistry, No.2(38), pp 108-112, (2005).

[3] Kobzhanov, A.S., Kurbatov, A.P., Romanov, G.A., Influence of electrolyte composition and temperature on the electro-deposition of copper powders., Industry of Kazakhstan, No.3(30), pp 80-81, (2005).

[4] Vargamyan, A.T., Electrodeposition of metals, Moscow, (1950).

[5] Patent 2126312 US, MPK $^{7}$ B22F009/24, C22B015/00, C25C001/12, C25D001/04 A method of producing metal powder, copper oxides and copper foil., David P. Byurgess, Wendy M. Gort, Ronald K. Haynes, Jackson G. Jenkins, Stephen J. Kohut, Peter Peckham, № 95119846; № 08/049160, (1994). 


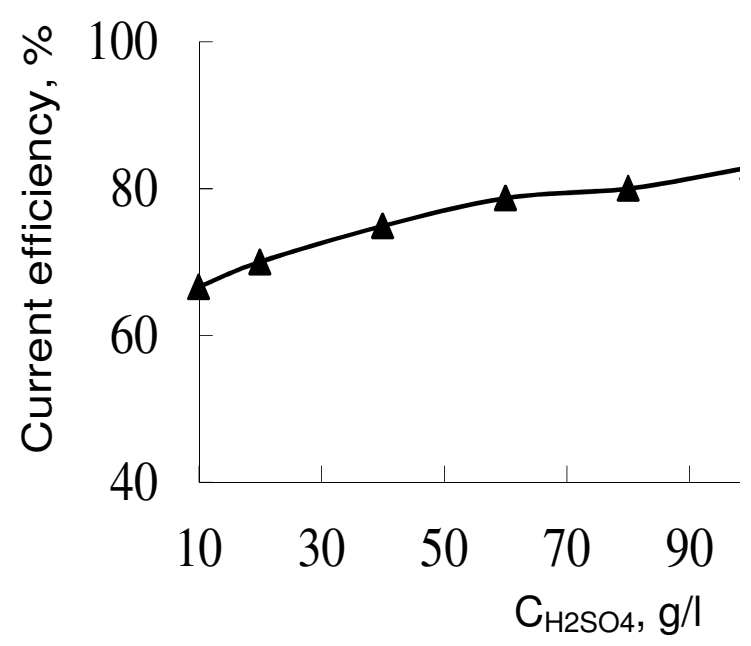

(a)

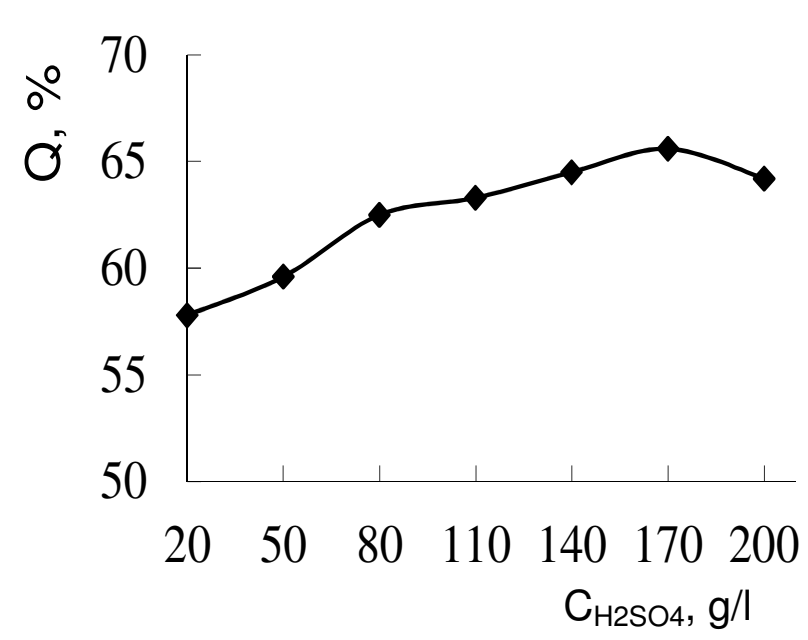

(b)

Fig.1. Effect of sulfuric acid concentration ( $\mathrm{C}_{\mathrm{H} 2 \mathrm{SO}}$ ) on copper current efficiency (a) and yield of fine fraction $(b)$ during the electrolysis using a copper cathode

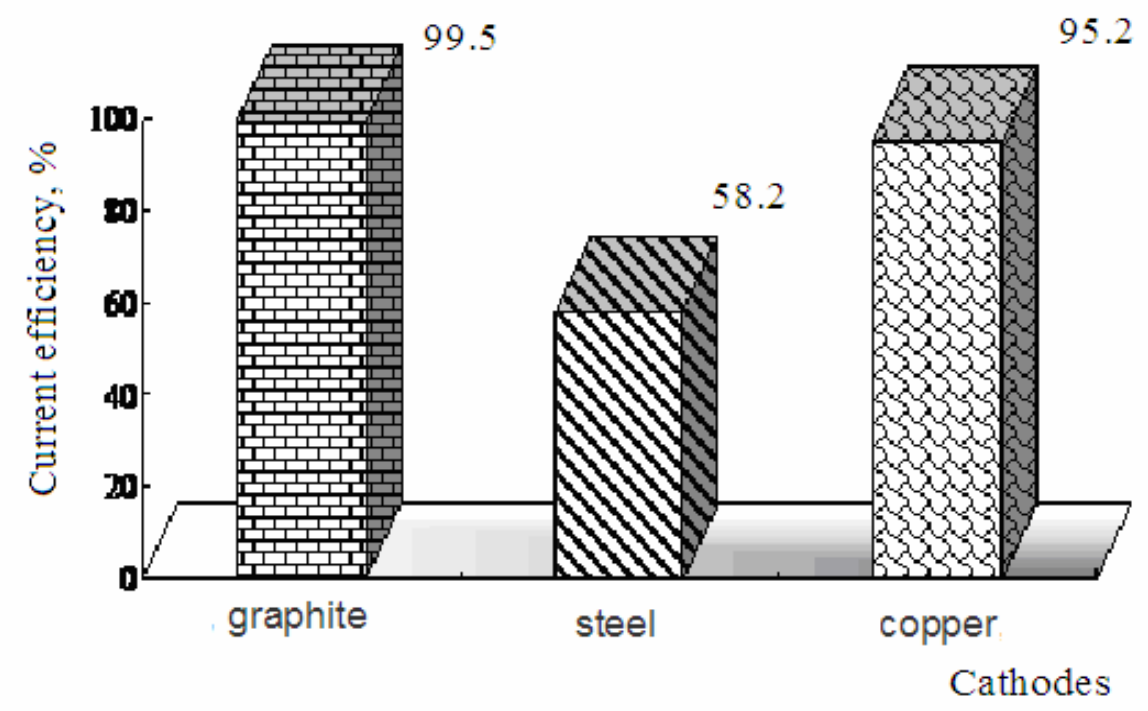

Fig.2. Effect of cathode material on the yield of the fine fraction of electrolytic copper powder 


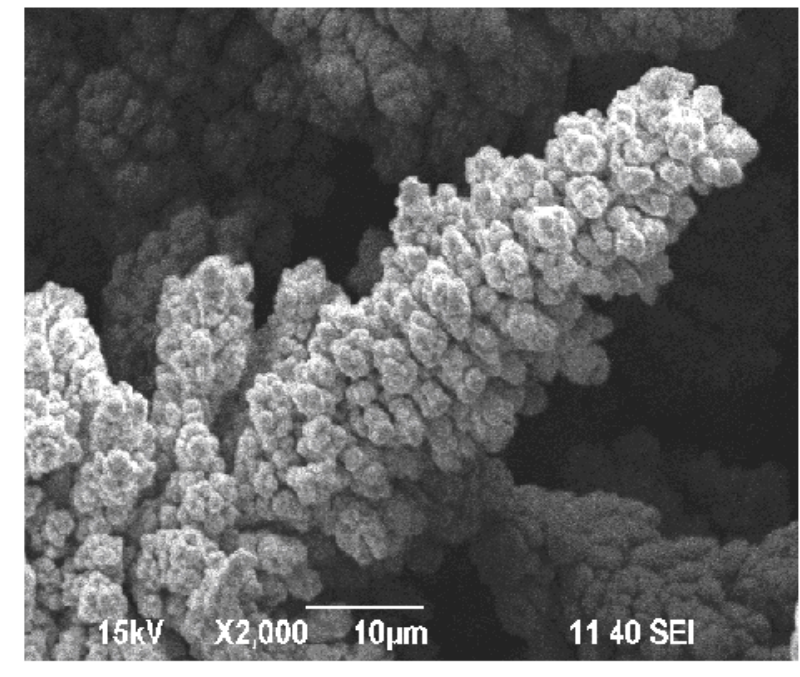

$C_{\mathrm{Cu}}=20 \mathrm{~g} / \mathrm{l} ; C_{\mathrm{H} 2 \mathrm{SO} 4}=50 \mathrm{~g} / \mathrm{l} ;$ electrolyte without organic additions

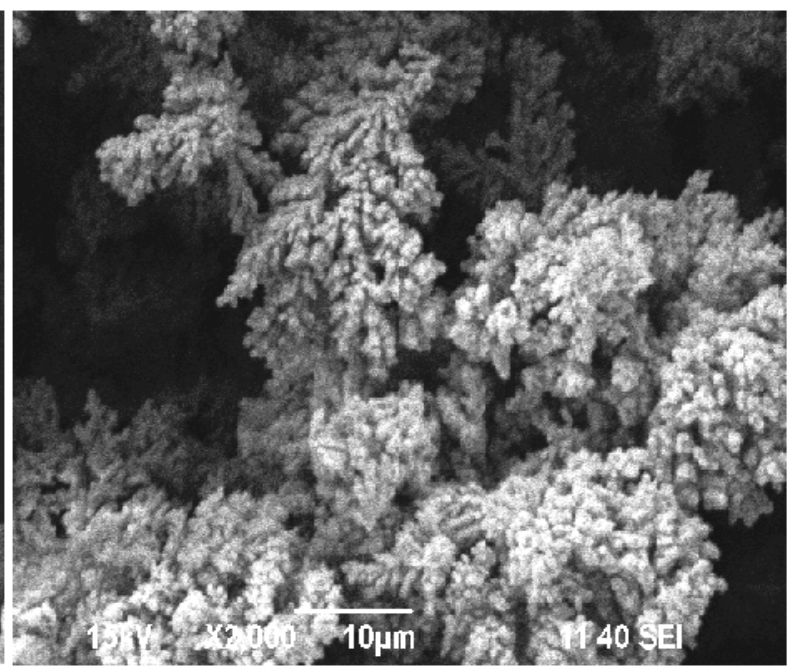

$C_{\mathrm{Cu}}=20 \mathrm{~g} / \mathrm{l} ; C_{\mathrm{H} 2 \mathrm{SO} 4}=50 \mathrm{~g} / \mathrm{l} ;$ addition of citric acid $0,1 \mathrm{~g} / \mathrm{l}$

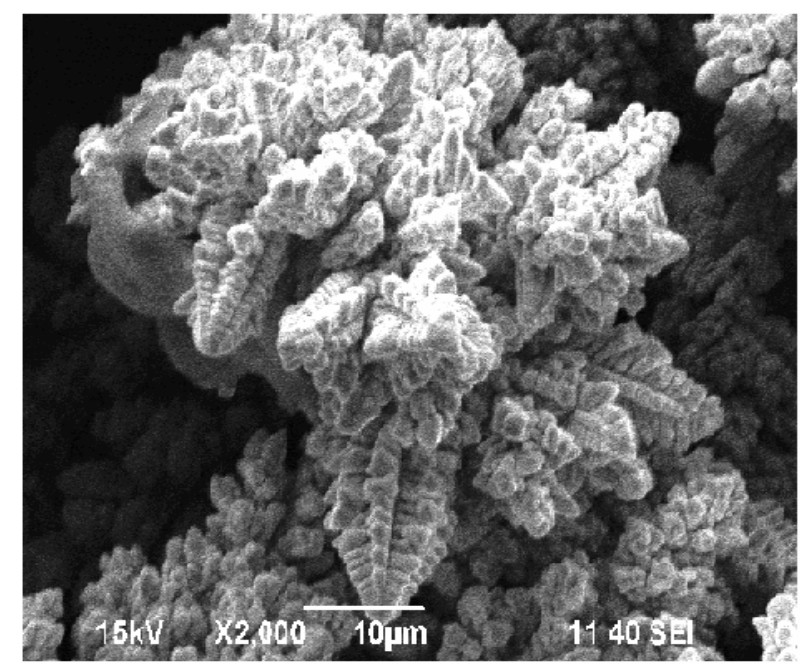

$C_{\mathrm{Cu}}=20 \mathrm{~g} / \mathrm{l} ; C_{\mathrm{H} 2 \mathrm{SO} 4}=50 \mathrm{~g} / \mathrm{l} ;$ addition of acetic acid $-2 \mathrm{ml} / \mathrm{l}$

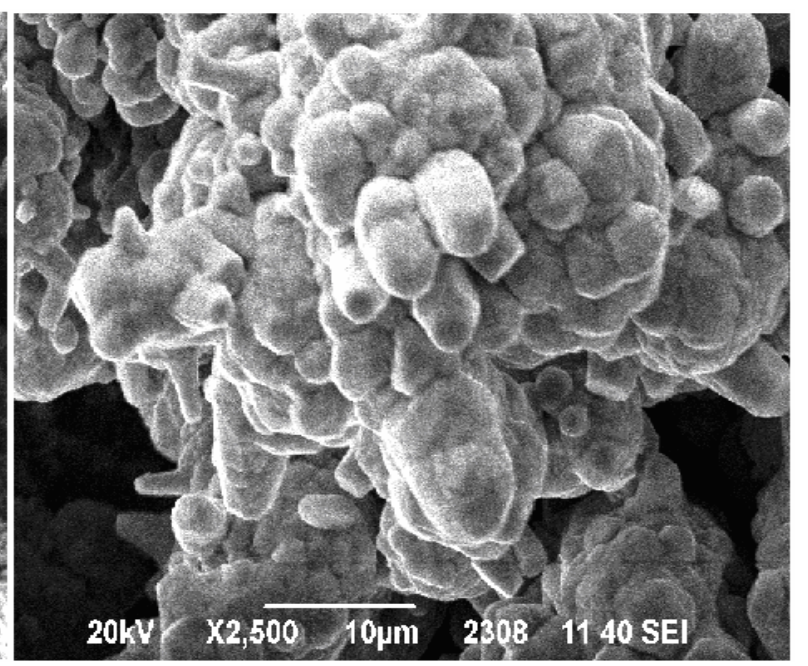

$C_{\mathrm{Cu}}=20 \mathrm{~g} / \mathrm{l} ; \mathrm{CH}_{\mathrm{H} 2 \mathrm{SO} 4}=50 \mathrm{~g} / \mathrm{l} ;$ addition of acetic acid $-20 \mathrm{ml} / \mathrm{l}$

Fig.3. Influence of organic compounds additions in the sulfate electrolyte on the shape and grain size of deposited copper powders 